HUPD-9923

hep-ph/0001321

\title{
CALCULATION OF DIRECT CP VIOLATION IN B DECAYS
}

\author{
Cai-Dian Lï̈ \\ Department of Physics, Hiroshima University, Higashi-Hiroshima 739-8526, \\ Japan \\ E-mail: lucd@theo.phys.sci.hiroshima-u.ac.jp
}

\begin{abstract}
Using the generalized factorization approach, we calculate the CP asymmetries of charmless B decays. A number of decay channels has large $\mathrm{CP}$ asymmetries, which can be measured in the $\mathrm{B}$ factories.
\end{abstract}

Invited talk given at the

3rd International Conference on B Physics and CP Violation (BCONF99)

Taipei, Taiwan, 3-7 December 1999

To appear in the Proceedings

${ }^{a}$ JSPS research fellow. 


\title{
CALCULATION OF DIRECT CP VIOLATION IN B DECAYS
}

\author{
Cai-Dian Lü \\ Department of Physics, Hiroshima University, Higashi-Hiroshima 739-8526, Japan \\ E-mail: lucd@theo.phys.sci.hiroshima-u.ac.jp
}

\begin{abstract}
Using the generalized factorization approach, we calculate the $\mathrm{CP}$ asymmetries of charmless B decays. A number of decay channels has large CP asymmetries, which can be measured in the $\mathrm{B}$ factories.
\end{abstract}

\section{Introduction}

The sourse of $\mathrm{CP}$ violation is one of the unsolved problem in the standard model (SM). The richness of charmless hadronic decays of B meson provides a good place for study of $\mathrm{CP}$ violation 1 . When $B^{0}$ and $\bar{B}^{0}$ decay to a common final state $f, B^{0}-\bar{B}^{0}$ mixing plays a crucial role. It interferences with the direct $\mathrm{CP}$ asymmetries. For other decays, $B^{0}$ and $\bar{B}^{0}$ decay to different final states, for example $B^{0} \rightarrow K^{+} \pi^{-}, \bar{B}^{0} \rightarrow K^{-} \pi^{+}$. No mixing is involved here. They are similar to charged $B^{ \pm}$decays. $\mathrm{CP}$ asymmetry has no time dependence. The direct $\mathrm{CP}$ asymmetry is important even if for neutral $\mathrm{B}$ meson decays.

If there is only one amplitude contributing to the decay, both the strong phase and weak phase can be factored out. we have $\Gamma=\bar{\Gamma}$. So there is no direct $\mathrm{CP}$ asymmetry. That is the case for $\mathrm{D}$ meson decays and $\mathrm{B}$ meson going to heavy meson decays, like $B \rightarrow J / \psi K_{S}$. If there are two amplitudes, the decay rate of $\Gamma$ and $\bar{\Gamma}$ may be different. If the strong phase difference between the two amplitudes $M_{1}$ and $M_{2}$ is not zero $\left(\delta_{12} \neq 0\right)$ and the weak phase difference of the two amplitudes is also non zero $\left(\phi_{12} \neq 0\right)$, we have $\Gamma \neq \bar{\Gamma}$. The direct CP asymmetry is

$$
A_{C P}=\frac{2 r \sin \delta_{12} \sin \phi_{12}}{1+r^{2}+2 r \cos \delta_{12} \cos \phi_{12}}
$$

where $r=\left|M_{2}\right| /\left|M_{1}\right| . A_{C P}$ depends on $2 r /\left(1+r^{2}\right), \sin \delta_{12}$ and $\sin \phi_{12}$. If one of the three parameters is small, then $A_{C P}$ is small. In many decays, we do not have all these conditions, then there is no sizable direct $\mathrm{CP}$ violation. However, most charmless decays have large values for $2 r /\left(1+r^{2}\right)$, where $M_{1}$ is tree amplitude and $M_{2}$ is penguin amplitude. Furthermore, the CKM parameters for the tree diagram and penguin diagrams are different providing weak phase differences.

Direct $\mathrm{CP}$ asymmetries require an interference between two amplitudes involving both a CKM phase and a final state strong interaction phase dif- 
ference. The weak phase difference arises from the superposition of penguin contributions and the tree diagrams. The strong-phase difference arises through the perturbative penguin diagrams (hard final state interaction), or non-perturbatively (soft final state interaction). The soft part is not important which is shown in some model calculations 2 . There are also some other contributions, such as annihilation diagram and Soft final state interaction. Mostly, their contributions to branching ratios are small 3 . Probably their contribution to $A_{C P}$ is also small. This is also shown in some model calculations 2 .

The method of Isospin or SU(3) symmetry ${ }^{3}$ which requires a set of measurements to solve the uncertainties is sometimes difficult for experiments. We estimate these strong phases in specific models, such like the generalized factorization approach, which can be tested by experiments.

\section{CP Violation Classification and Formulae}

For charged $B^{ \pm}$decays the CP-violating asymmetries are defined as 1

$$
A_{C P}=\frac{\Gamma\left(B^{+} \rightarrow f^{+}\right)-\Gamma\left(B^{-} \rightarrow f^{-}\right)}{\Gamma\left(B^{+} \rightarrow f^{+}\right)+\Gamma\left(B^{-} \rightarrow f^{-}\right)} .
$$

The charged modes are self-tagged decay channels for experiments. They are easy to be measured. For $B^{0}$ decays, more complication is from the $B^{0}-\bar{B}^{0}$ mixing. The CP-asymmetries may be time-dependent, if the final states are the same for $B^{0}$ and $\overline{B^{0}}$

$$
\begin{aligned}
A_{C P}(t) & =\frac{\Gamma\left(B^{0}(t) \rightarrow f\right)-\Gamma\left(\bar{B}^{0}(t) \rightarrow f\right)}{\Gamma\left(B^{0}(t) \rightarrow f\right)+\Gamma\left(\bar{B}^{0}(t) \rightarrow f\right)} \\
& \simeq a_{\epsilon^{\prime}} \cos (\Delta m t)+a_{\epsilon+\epsilon^{\prime}} \sin (\Delta m t) .
\end{aligned}
$$

Here the direct $\mathrm{CP}$ violation parameter $a_{\epsilon^{\prime}}$ is defined as

$$
a_{\epsilon^{\prime}}=A_{C P}^{d i r}=\frac{\Gamma\left(B^{0} \rightarrow f\right)-\Gamma\left(\bar{B}^{0} \rightarrow f\right)}{\Gamma\left(B^{0} \rightarrow f\right)+\Gamma\left(\bar{B}^{0} \rightarrow f\right)},
$$

which is the same defination as the charged B decays. And $a_{\epsilon+\epsilon^{\prime}}$ is mixinginduced $\mathrm{CP}$ violation 1 . In this note we will concentrate on the direct $\mathrm{CP}$ asymmetries. 


\section{$2.1 b \rightarrow s(\bar{b} \rightarrow \bar{s})$, transitions}

First we parametrize the decay amplitude like this way ${ }^{\mathrm{a}}$

$$
\begin{aligned}
& \mathcal{M}=T \xi_{u}-P_{t} \xi_{t} e^{i \delta_{t}}-P_{c} \xi_{c} e^{i \delta_{c}}-P_{u} \xi_{u} e^{i \delta_{u}}, \\
& \overline{\mathcal{M}}=T \xi_{u}^{*}-P_{t} \xi_{t}^{*} e^{i \delta_{t}}-P_{c} \xi_{c}^{*} e^{i \delta_{c}}-P_{u} \xi_{u}^{*} e^{i \delta_{u}},
\end{aligned}
$$

where $\xi_{i}=V_{i b} V_{i s}^{*}$. T and $P_{i}$ are the tree and $i(i=u, c, t)$ quark penguin contributions, respectively. Working in SM, we can use the unitarity relation $\xi_{c}=-\xi_{u}-\xi_{t}$ to simplify the above equation,

$$
\begin{aligned}
& \mathcal{M}=T \xi_{u}-P_{t c} \xi_{t} e^{i \delta_{t c}}-P_{u c} \xi_{u} e^{i \delta_{u c}} \\
& \overline{\mathcal{M}}=T \xi_{u}^{*}-P_{t c} \xi_{t}^{*} e^{i \delta_{t c}}-P_{u c} \xi_{u}^{*} e^{i \delta_{u c}}
\end{aligned}
$$

where we define

$$
\begin{aligned}
P_{t c} e^{i \delta_{t c}} & =P_{t} e^{i \delta_{t}}-P_{c} e^{i \delta_{c}}, \\
P_{u c} e^{i \delta_{u c}} & =P_{u} e^{i \delta_{u}}-P_{c} e^{i \delta_{c}} .
\end{aligned}
$$

Thus, the direct CP-violating asymmetry is

$$
A_{C P} \equiv a_{\epsilon^{\prime}}=\left(|\overline{\mathcal{M}}|^{2}-|\mathcal{M}|^{2}\right) /\left(|\mathcal{M}|^{2}+|\overline{\mathcal{M}}|^{2}\right)=A^{-} / A^{+},
$$

where

$$
\begin{aligned}
A^{-}= & 2 T P_{t c}\left|\xi_{u}^{*} \xi_{t}\right| \sin \phi_{3} \sin \delta_{t c}+2 P_{t c} P_{u c}\left|\xi_{u}^{*} \xi_{t}\right| \sin \phi_{3} \sin \left(\delta_{u c}-\delta_{t c}\right), \\
A^{+}= & \left(T^{2}+P_{u c}^{2}\right)\left|\xi_{u}\right|^{2}+P_{t c}^{2}\left|\xi_{t}\right|^{2}-2 P_{t c} P_{u c}\left|\xi_{u}^{*} \xi_{t}\right| \cos \phi_{3} \cos \left(\delta_{u c}-\delta_{t c}\right) \\
& -2 T P_{u c}\left|\xi_{u}\right|^{2} \cos \delta_{u c}+2 T P_{t c}\left|\xi_{u}^{*} \xi_{t}\right| \cos \phi_{3} \cos \delta_{t c} .
\end{aligned}
$$

First, we note that $\left|\xi_{u}\right| \ll\left|\xi_{t}\right| \simeq\left|\xi_{c}\right|$, with an upper bound $\left|\xi_{u}\right| /\left|\xi_{t}\right| \leq$ 0.025. In some channels, such as $B^{+} \rightarrow K^{+} \pi^{0}, K^{*+} \pi^{0}, K^{*+} \rho^{0}, B^{0} \rightarrow K^{+} \pi^{-}$, $K^{*+} \pi^{-}, K^{*+} \rho^{-},\left|P_{t c} / T\right|$ is of $O(0.1),\left|P_{u c} / P_{t c}\right|=O(0.3)$. The CP-violating asymmetry in this case is

$$
A_{C P} \simeq \frac{2 z_{12} \sin \delta_{t c} \sin \phi_{3}}{1+2 z_{12} \cos \delta_{t c} \cos \phi_{3}+z_{12}^{2}},
$$

where $z_{12}=\left|\xi_{u} / \xi_{t}\right| \times T / P_{t c}$. We show the CP asymmetry of $B \rightarrow K^{*+} \pi^{-}$ as an example in Figure 1. It is easy to see that, there may be large $\mathrm{CP}$ asymmetries in this decay channel. Besides the CKM parameter $\rho$ and $\eta$, the $\mathrm{CP}$ asymmetry is also sensitive to the gluon momentum $k^{2}$, which is related 


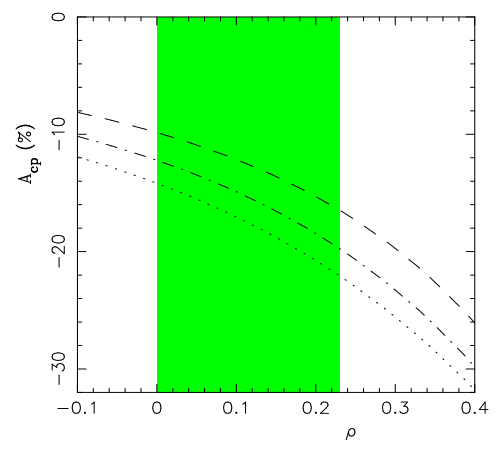

(a)

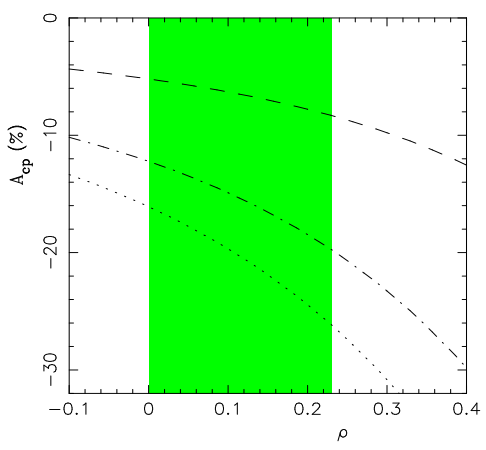

(b)

Figure 1: CP-violating Asymmetry $A_{C P}$ in $B^{0} \rightarrow K^{*+} \pi^{-}$decay as a function of the CKM parameter $\rho$. (a) $k^{2}=m_{b}^{2} / 2$. The dotted, dashed-dotted and dashed curves correspond to the CKM parameter values $\eta=0.42, \eta=0.34$ and $\eta=0.26$, respectively. (b) $\eta=0.34$. The dotted, dashed-dotted and dashed curves correspond to $k^{2}=m_{b}^{2} / 2+2 \mathrm{GeV}^{2}, k^{2}=m_{b}^{2} / 2$ and $k^{2}=m_{b}^{2} / 2-2 \mathrm{GeV}^{2}$, respectively.

to the size of strong phase. If $k^{2}$ is known, the strong phase is predictable, we may use $A_{C P}$ to determine $\sin \phi_{3}$. The first 6 channels of Table 1 are this kind of decays. Two of them are reported from CLEO Collaboration with large error-bars $\mathrm{E}^{\mathrm{B}}$. The central values are far away from the theoretical predictions. If more data suport this, we may expect new physics signals here.

There are some decays with vanishing tree contributions $(T=0)$, such as $B^{+} \rightarrow \pi^{+} K_{S}^{0}, \pi^{+} K^{* 0}, \rho^{+} K^{* 0}$. Then for these decays, the CP-violating asymmetry is

$$
A_{C P} \simeq 2 \frac{P_{u c}}{P_{t c}}\left|\frac{\xi_{u}}{\xi_{t}}\right| \sin \left(\delta_{u c}-\delta_{t c}\right) \sin \phi_{3} .
$$

Without the tree contribution, the suppression due to both $P_{u c} / P_{t c}$ and $\left|\xi_{u} / \xi_{t}\right|$ is much stronger. The CP-violating asymmetries are only around $-(1-2) \%$.

Some estimates of the channel $B^{+} \rightarrow \pi K_{S}^{0}$ show that even including the annihilation and soft final state interaction, the CP asymmetry of this decay is still small2. This means that this channel is clean for new physics to show up. In table 1, we can see that CLEO's central value of this decay indicates a large $\mathrm{CP}$ asymmetry maybe possible.

\section{2 $b \rightarrow d(\bar{b} \rightarrow \bar{d})$ transitions}

Similarly to the $b \rightarrow s$ transition, we can define the CP asymmetry as

$$
A_{C P}=A^{-} / A^{+},
$$


Table 1: CP-rate asymmetries $A_{C P}$ and branching ratios for some $B \rightarrow h_{1} h_{2}$ decays, updated for the central values of the CKM fits $\rho=0.20, \eta=0.37$ and the factorization model parameters $\xi=0.5$ and $k^{2}=m_{b}^{2} / 2 \pm 2 \mathrm{GeV}^{2}$.

\begin{tabular}{|c|c|c|l|}
\hline Decay Modes & $A_{C P}$-Exp.(\%) & $A_{C P}(\%)$ & $B R\left(\times 10^{-6}\right)$ \\
\hline$B^{ \pm} \rightarrow K^{ \pm} \pi^{0}$ & $29 \pm 23$ & $-7.7_{+4.0}^{-2.2}$ & 10.0 \\
\hline$B^{ \pm} \rightarrow K^{* \pm} \pi^{0}$ & - & $-14.4_{+8.2}^{-4.4}$ & 4.3 \\
\hline$B^{ \pm} \rightarrow K^{* \pm} \rho^{0}$ & - & $-13.5_{+7.5}^{-4.0}$ & 4.8 \\
\hline$B^{0} \rightarrow K^{+} \pi^{-}$ & $4 \pm 16$ & $-8.2_{+4.3}^{-2.3}$ & 14.0 \\
\hline$B^{0} \rightarrow K^{*+} \pi^{-}$ & - & $-17.2_{+9.8}^{-5.5}$ & 6.0 \\
\hline$B^{0} \rightarrow K^{*+} \rho^{-}$ & - & $-17.2_{+9.8}^{-5.5}$ & 5.4 \\
\hline$B^{ \pm} \rightarrow K_{S}^{0} \pi^{ \pm}$ & $-18 \pm 24$ & $-1.4_{++.1}^{-0.1}$ & 14.0 \\
\hline$B^{ \pm} \rightarrow \eta \pi^{ \pm}$ & - & $9.3_{-4.1}^{+1.9}$ & 5.5 \\
\hline$B^{ \pm} \rightarrow \eta^{\prime} \pi^{ \pm}$ & - & $9.4_{-4.5}^{+2.1}$ & 3.7 \\
\hline$B^{ \pm} \rightarrow \eta \rho^{ \pm}$ & - & $3.1_{-1.7}^{+0.7}$ & 8.6 \\
\hline$B^{ \pm} \rightarrow \eta^{\prime} \rho^{ \pm}$ & - & $3.1_{-1.8}^{+0.7}$ & 6.2 \\
\hline$B^{ \pm} \rightarrow \rho^{ \pm} \omega$ & - & $7.0_{-3.4}^{+1.5}$ & 21.0 \\
\hline$B^{ \pm} \rightarrow \eta^{\prime} K^{ \pm}$ & $-3 \pm 12$ & $-4.9_{+2.1}^{-1.2}$ & 23.0 \\
\hline$B^{ \pm} \rightarrow \pi^{ \pm} \omega$ & $34 \pm 25$ & $7.7_{-3.7}^{+1.7}$ & 9.5 \\
\hline
\end{tabular}

where

$$
\begin{aligned}
A^{-}= & -2 T P_{t c}\left|\zeta_{u}^{*} \zeta_{t}\right| \sin \phi_{2} \sin \delta_{t c}-2 P_{t c} P_{u c}\left|\zeta_{u}^{*} \zeta_{t}\right| \sin \phi_{2} \sin \left(\delta_{u c}-\delta_{t c}\right), \\
A^{+}= & \left(T^{2}+P_{u c}^{2}\right)\left|\zeta_{u}\right|^{2}+P_{t c}^{2}\left|\zeta_{t}\right|^{2}-2 P_{t c} P_{u c}\left|\zeta_{u}^{*} \zeta_{t}\right| \cos \phi_{2} \cos \left(\delta_{u c}-\delta_{t c}\right) \\
& -2 T P_{u c}\left|\zeta_{u}\right|^{2} \cos \delta_{u c}+2 T P_{t c}\left|\zeta_{u}^{*} \zeta_{t}\right| \cos \phi_{2} \cos \delta_{t c}
\end{aligned}
$$

with $\zeta_{i}=V_{i b} V_{i d}^{*}$, and again we have used CKM unitarity relation $\zeta_{c}=-\zeta_{t}-\zeta_{u}$.

For the tree-dominated decays, such as $B^{+} \rightarrow \pi^{+} \eta^{(\prime)}, \rho^{+} \eta^{(\prime)}, \rho^{+} \omega$, the relation $P_{u c}<P_{t c} \ll T$ holds. The CP asymmetry is

$$
A_{C P} \simeq \frac{-2 z_{1} \sin \delta_{t c} \sin \phi_{2}}{1+2 z_{1} \cos \delta_{t c} \cos \phi_{2}}
$$

with $z_{1}=\left|\zeta_{t} / \zeta_{u}\right| \times T P_{t c} / T^{\prime 2}$, and $T^{\prime 2} \equiv T^{2}-2 T P_{u c} \cos \delta_{u c}$. The CP asymmetries are proportional to $\sin \phi_{2}$. They are large enough for the experiments to detect them. The theoretical predictions of these decays are shown in table 1.

For the decays with a vanishing tree contribution $(T=0)$, such as $B^{+} \rightarrow$ $K^{+} K_{S}^{0}, K^{+} \bar{K}^{* 0}, K^{*+} \bar{K}^{* 0}$, the CP-violating asymmetry is approximately pro- 
portional to $\sin \phi_{2}$ again,

$$
A_{C P}=\frac{-2 z_{3} \sin \left(\delta_{u c}-\delta_{t c}\right) \sin \phi_{2}}{1-2 z_{3} \cos \left(\delta_{u c}-\delta_{t c}\right) \cos \phi_{2}+z_{3}^{2}},
$$

with $z_{3}=\left|\zeta_{u} / \zeta_{t}\right| \times P_{u c} / P_{t c}$. As the suppressions from $\left|\zeta_{u} / \zeta_{t}\right|$ and $\left|P_{u c} / P_{t c}\right|$ are not very big, the CP-violating asymmetry can again be of prder (10-20)\%. Unfortunately, these channels have smaller branching ratios 4 .

More charmless decay channels are discussed in reft. Some of them are more complicated than the ones we discussed above. There are also some other interesting decays like $B \rightarrow K^{*} \gamma, B \rightarrow D \pi \ell \nu, B \rightarrow \pi \pi \ell \nu$ G, etc. They have small CP asymmetries in SM. They are sensitive to new physics.

\section{Models of Calculation}

In the Factorization Approach 1 , the two body $B$ meson decays can be factorized as two products:

$$
C_{i}\left\langle P_{1} P_{2}\left|O_{i}\right| B\right\rangle=C_{i}\left\langle P_{1}\left|J_{\mu}\right| 0\right\rangle\left\langle P_{2}\left|J^{\mu}\right| B\right\rangle,
$$

where $C_{i}$ is the corresponding Wilson coefficients. The second factor on the right side of the equation is proportional to the meson decay constant. The last term is the corresponding form factors.

The strong-phase differences arise through Bander-Silverman-Soni Mechanism (BSS) 1 . In this picture, the perturbative penguin diagrams involving charm and up quark loops, where the light quarks can be on mass shell, providing the strong phases. They are mostly sensitive to the gluon momentum $k^{2}$. For numerical calculations, we use $k^{2}=m_{b}^{2} / 2 \neq 2 \mathrm{GeV}^{2}$.

In the perturbative QCD approach (pQCD) 9 , we need one hard gluon connecting the spectator quark. Strong phases are from the non-factorizable diagram and annihilation diagram, where the innner quark or gluon propagator can be on mass shell. The pQCD approach is based on factorization, and goes one step further. In pQCD, we can calculate annihilation diagrams and also the non-factorizable contributions. The $k^{2}$ of gluon is well defined in this approach. We have calculated the $B \rightarrow \pi \pi$ decays in this approach, and the results compared with the factorization approach in Table 2 .

\section{Summary}

The recently measured direct $\mathrm{CP}$ asymmetries for $B^{0} \rightarrow K^{+} \pi^{-}, B^{+} \rightarrow K^{+} \eta^{\prime}$, $B^{0} \rightarrow K^{+} \pi^{-}, B^{+} \rightarrow \pi^{+} K^{0}$, and $B^{+} \rightarrow \omega \pi^{+}$are encouraging news for direct $\mathrm{CP}$ violation in $\mathrm{B}$ decays, although the signal is not significantly excess background. 
Table 2: Direct CP asymmetries of $\beta \rightarrow \pi \pi$ decays in factorization approach and perturbative QCD approach (Preliminary) 10 .

\begin{tabular}{|c|c|c|}
\hline Channel & $A_{C P}^{\text {direct }}$ (Factorization) & $A_{C P}^{\text {direct }}(\mathrm{pQCD})$ \\
\hline$B \rightarrow \pi^{+} \pi^{-}$ & $6.9 \%_{-4}^{+2}$ & $-1.4 \%$ \\
\hline$\overline{B^{+} \rightarrow \pi^{+} \pi^{0}}$ & $0.1 \%_{-0.1}^{+0.1}$ & $0.02 \%$ \\
\hline$B^{0} \rightarrow \pi^{0} \pi^{0}$ & $\begin{array}{l}-15 \%+14 \\
-7\end{array}$ & $-60 \%$ \\
\hline
\end{tabular}

CP-asymmetries of $A_{C P}\left(K^{ \pm} \eta^{\prime}\right), A_{C P}\left(\pi^{ \pm} K_{S}^{0}\right)$ and $A_{C P}\left(\rho^{ \pm} \stackrel{(-)}{K^{* 0}}\right)$ are small, but stable against variation in $N_{c}, k^{2}$ and $\mu$. CP-asymmetries well over $10 \%$ in these decay modes will be a sign of new physics. The decay channels of $B \rightarrow K^{* \pm} \pi^{\mp}, K^{* \pm} \pi^{0}, K^{* \pm} \eta, K^{* \pm} \eta^{\prime}, K^{* \pm} \rho^{\mp}$ and $K^{* \pm} \rho^{0}$, have measurably large $\mathrm{CP}$-violating asymmetries. A good measurement of the CP-asymmetry in any one of these decays could be used to determine $k^{2}$. Such that the theoretical predictions of all other channels make sense. We also hope that the perturbative QCD approach could solve the remaining uncertainties in the factorization approach. With the two B factories and other hadronic machines, a number of decays is going to be measured soon.

\section{Acknowledgments}

The author thanks A. Ali and G. Kramer for collaboration on the main topic discussed in this report. We thank the organizer H.Y. Cheng and W.S. Hou for a fruitful BCP3 conference. We acknowledge the Grant-in-Aid for Scientific Research on Priority Areas (Physics of CP violation) and JSPS for support.

\section{References}

1. A. Ali, G. Kramer, C.D. Lü, Phys. Rev. D59, 014005 (1999).

2. H.Y. Jin, hep-ph/9805235; D.S. Du, X.Q. Li, Z.T. Wei, B.S. Zou, Eur. Phys. J. A4, 91 (1999); M. Neubert, Phys. Lett. B424, 152 (1998); A,N. Kamal, Phys. Rev. D60, 094018 (1999).

3. A. Ali, G. Kramer, C.D. Lü, Phys. Rev. D58, 094009 (1998); C.D. Lü, Nucl. Phys. Proc. Suppl. 74, 227 (1999).

4. M. Gronau, in these proceedings.

5. S. Chen et al., CLEO Collaboration, hep-ex/0001009.

6. C.S. Kim, J. Lee, W. Nagung, Phys. Rev. D60, 094019 (1999); Phys. Rev. D59, 114006 (1999); Phys. Rev. D59, 114005 (1999).

7. H.Y. Cheng, in these proceedings, hep-ph/9912372.

8. M. Bander, D. Silverman, A. Soni, Phys. Rev. Lett. 43, 242 (1979). 
9. H.N. Li, in these proceedings.

10. C.D. Lü, K. Ukai, M.Z. Yang, in progress. 